\title{
Phase Transition to Hyperon Matter in Neutron Stars
}

\author{
Jürgen Schaffner-Bielich \\ Department of Physics, Columbia University, 538 West 120th Street, New York, NY 10027, USA \\ Matthias Hanauske, Horst Stöcker, and Walter Greiner \\ Institut für Theoretische Physik, J. W. Goethe-Universität, D-60054 Frankfurt am Main, Germany
}

(October 1, 2005)

Recent progress in the understanding of the high density phase of neutron stars advances the view that a substantial fraction of the matter consists of hyperons. The possible impacts of a highly attractive interaction between hyperons on the properties of compact stars are investigated. We find that a hadronic equation of state with hyperons allows for a first order phase transition to hyperonic matter. The corresponding hyperon stars can have rather small radii of $R \approx 8 \mathrm{~km}$.

PACS: $26.60+\mathrm{c}, 21.65+\mathrm{f}, 97.60 . \mathrm{Gb}, 97.60 . \mathrm{Jd}$

Neutron stars are an excellent observatory to probe our understanding of the theory of strongly interacting matter at extreme densities. The interior of neutron stars is dense enough to allow for the appearance of new particles with the quantum number strangeness besides the conventional nucleons and leptons by virtue of weak equilibrium. There is growing support that hyperons are the first exotic particle to appear in neutron star matter at around twice normal nuclear density [1] , as recently confirmed within various different models as effective nonrelativistic potential models [2, the Quark-Meson Coupling Model [3], extended Relativistic Mean-Field approaches [4.5], Relativistic Hartree-Fock [6], Brueckner-HartreeFock [7,8], and chiral effective Lagrangians [9]. The onset of hyperon formation is controlled by the attractive hyperon-nucleon interaction as extracted from hyperonnucleon scattering data and hypernuclear data. The hyperon population rapidly increases above the critical density, eventually even exceeding that of the nucleons. The question arises to what extent does the interaction between the hyperons, which is essentially unknown, influence the overall properties of the compact star.

In this Letter, we will demonstrate that a first order phase transition to strange hadronic matter due to highly attractive hyperon-hyperon interactions can occur which drastically changes the global features of neutron stars and leads to compact stars with unusually small radii of $R \approx 8 \mathrm{~km}$. Simultaneous mass and radius measurements of a neutron star could reveal or rule out the existence of such a novel form of matter with exotic properties, which is in accord with our present knowledge of hadronic physics. It is this enormous number of hyperons in neutron stars which enables the formation of such exotic compact stars with strangeness.
Nuclear systems with strangeness, hypernuclei, have been studied in the last decades both experimentally and theoretically. From these studies we know that the nucleon- $\Lambda$ interaction is attractive and that the $\Lambda$ feels a potential of about $U_{\Lambda}=-28 \mathrm{MeV}$ in bulk matter [10]. On the other hand, extrapolated $\Sigma^{-}$atomic data indicate that the isoscalar potential is repulsive in the nuclear core 111] which is supported by the absence of bound states in a recent $\Sigma$-hypernuclear search [12]. An attractive potential for the double strange hyperon $\Xi$ has been extracted from the few $\Xi$ hypernuclear events 13 and indirectly from final state interactions at KEK 14 and at Brookhaven's AGS [15]. Recently, double $\Lambda$ hypernuclear events have been reported by E906 at AGS [16] and E373 at KEK [17] in addition to the older hypernuclear events. The $\Lambda \Lambda$ interaction as deduced from these double $\Lambda$ hypernuclear data is highly attractive (see [18] and references therein). There is no experimental information about the other hyperon-hyperon interactions, such as e.g. $\Lambda \Xi$ and $\Xi \Xi$ interactions.

A recent version of the Nijmegen soft-core potential finds extremely attractive hyperon-hyperon interactions which even allows for the possibility of deeply bound states of two hyperons [19] and deeply bound hyperonic matter 20]. Strange hadronic matter in general will consist of nucleons and arbitrary numbers of the hyperons $\Lambda, \Sigma, \Xi$, and $\Omega^{-}$. If the hyperon-hyperon interaction is only slightly attractive, strange hadronic matter in bulk is bound and purely hyperonic nuclei (MEMO's) are predicted to exist [21]. The driving force is the Pauliblocking in the hyperon world, which forbids $\Xi$ 's to decay to $\Lambda$ 's. Strange hadronic matter is metastable, i.e. it decays on the timescale of the hyperon weak decay of $\tau \approx 10^{-10} \mathrm{~s}$ by loosing one unit of strangeness. This short-lived exotic matter can be formed in relativistic heavy ion collisions 22], as hyperons are copiously produced in a single central event. Neutron star matter, however, is in $\beta$-equilibrium so that hyperon matter in neutron stars is stable on astrophysical timescales.

In the following, we choose the standard nuclear field theory of baryons interacting with mesons, which is solved in the mean-field approximation [1] and has been successfully applied to describe hypernuclear data [23]. The model is extended in a controlled fashion to include the baryon octet coupled to the full nonets of scalar and vector mesons [21,5,20] and is then extrapolated to large 


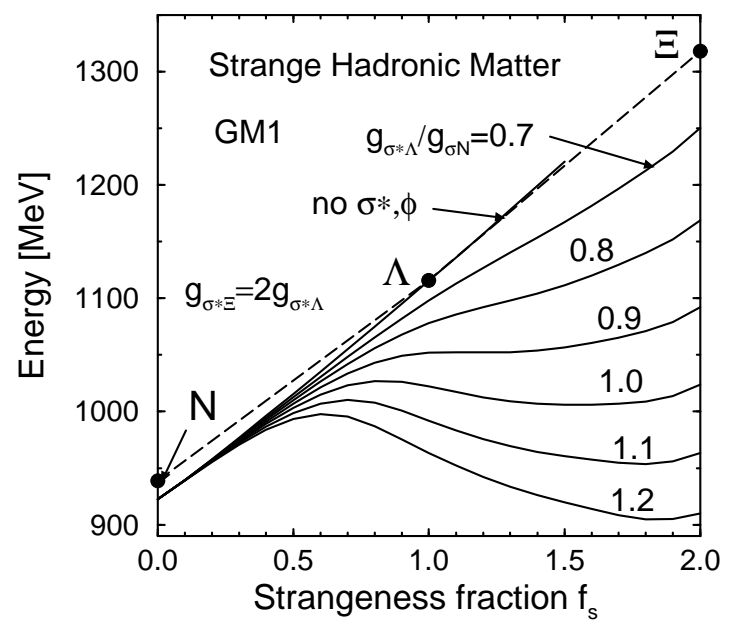

FIG. 1. Equation of state of strange hadronic matter for different strengths of the hyperon-hyperon interactions. A second stable minimum appears at large strangeness fraction $f_{s}$ which can be deeper than ordinary matter.

densities. The baryon-baryon interactions are mediated by scalar meson, $\sigma$, and vector meson, $\omega$, and isovector meson, $\rho$, exchange. In addition, hyperon-hyperon interactions are modeled via hidden strange meson exchange of a scalar, $\sigma^{*}$, and a vector, $\phi$, meson. The $\sigma^{*}$ and $\phi$ mesons couple to hyperons only. We take the nucleon parameterization from Glendenning and Moszkowski [24]. The coupling constants of the hyperons to the $\omega, \rho, \phi$ vector mesons are fixed by using $\mathrm{SU}(6)$ symmetry. The coupling constants to the $\sigma$ meson are constrained by the hypernuclear potential in nuclear matter of $U_{\Lambda}=-28$ $\mathrm{MeV}, U_{\Sigma}=+30 \mathrm{MeV}, U_{\Xi}=-18 \mathrm{MeV}$ to be compatible with hypernuclear data 20]. The remaining coupling constants of the hyperons to the $\sigma^{*}$ meson are varied to investigate the effects of an enhanced hyperon-hyperon interaction as suggested by the sparse $\Lambda \Lambda$ data. We allow these coupling constants to scale with the number of strange quarks of the hyperon. The coupling constant of the $\Lambda$ hyperon to the $\sigma^{*}$ meson is taken close to the corresponding nucleon $\sigma$ meson coupling constant $g_{\sigma N}$.

First, we discuss the stability of strange hadronic matter in bulk relevant for heavy ion physics. Figure 1 shows the total energy per baryon as a function of the strangeness fraction $f_{S}$, i.e. the number of strange quarks per baryon. The dashed line denotes the border between bound and unbound strange hadronic matter. Even in the absence of the hidden strange meson exchange, strange hadronic matter is bound up to $f_{S} \approx 1.5$. If the hyperon-hyperon interaction is taken into account, the matter gets more deeply bound at large $f_{S}$. Note that the curves for $f_{S} \lesssim 0.4$ hardly change and are compatible with hypernuclear data which probe at most $f_{S} \leq 1 / 3$, i.e. for the lightest hypernucleus ${ }_{\Lambda}^{3} \mathrm{H}$. For $g_{\sigma^{*}} / g_{\sigma N}>0.9$, a local second minimum appears at large $f_{S}>1$. This second minimum has been also seen in an effective param-

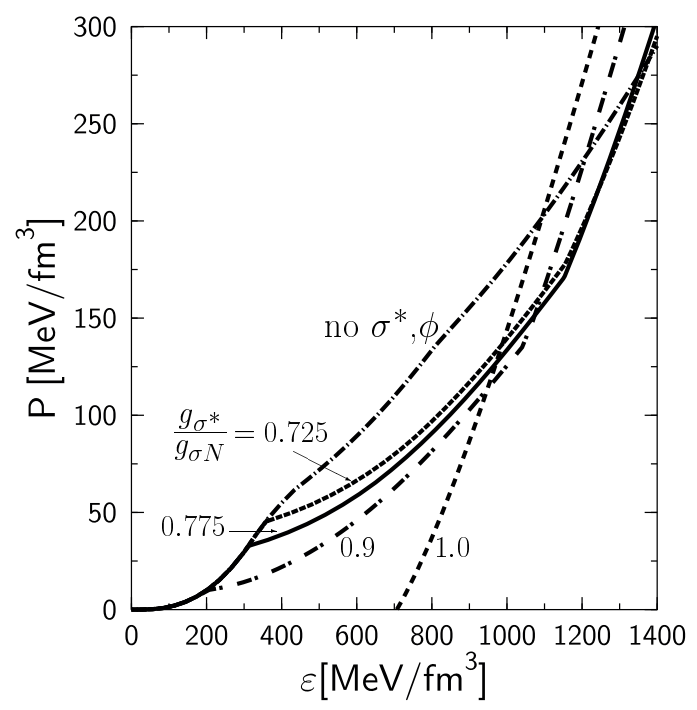

FIG. 2. Equation of state in $\beta$ equilibrium (neutron star matter) for different strengths of the hyperon-hyperon interactions.

eterization of the recent Nijmegen model [20]. Matter in this minimum is long-lived as it can only decay into nucleons through a multiple weak decay. The minimum is shifted below the nucleon mass for even larger values of $g_{\sigma^{*}} / g_{\sigma N} \geq 1.2$, thus creating absolutely stable strange hadronic matter 25. The collapse of nuclei into this absolutely stable form is prohibited, as it would violate strangeness conservation.

Let us discuss now the possible implications of deeply bound hyperonic matter for compact astrophysical objects. The equation of state (EoS) for charge neutral $\beta$-equilibrated neutron star matter is plotted in Fig. 2 . A first order phase transition to hypermatter appears which is seen as a pronounced softening of the EoS. The two kinks in the EoS mark the beginning and the end of the mixed phase where normal and hyperonic matter are coexisting. The critical energy density for the onset of the mixed phase region is lowered for stronger hyperonhyperon interactions. If a second minimum is present for strange hadronic matter $\left(g_{\sigma^{*}} / g_{\sigma N} \geq 1.0\right.$, see Fig. 11), the EoS exhibits a finite value of the energy density even for vanishing pressure, indicating that hypermatter becomes self-bound. The corresponding compact star is then bound by the interaction not by gravity. We stress that strange hadronic matter in the model does not need to be absolutely stable to produce self-bound compact stars. The presence of a second minimum, be it meta stable or absolutely stable, seems to be sufficient to generate self-bound hyperon stars!

The global feature of the neutron star changes drastically when the hyperon-hyperon interaction is switched on, even for small hyperon coupling constants (see Fig. 3). Without hidden strange meson exchange we find a maximum mass of $M_{\max }=1.8 M_{\odot}$ with a min- 


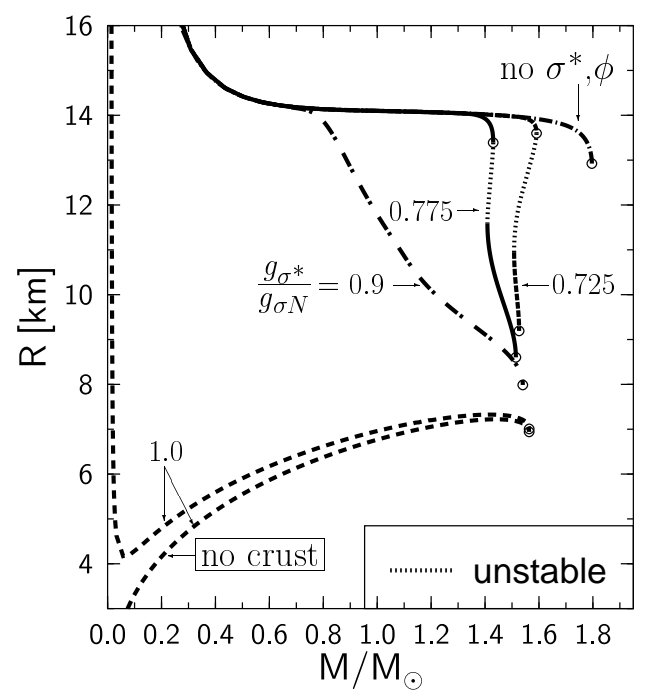

FIG. 3. Mass-radius relation for neutron stars with a highly attractive hyperon-hyperon interaction.

imum radius of $R_{\min }=12.9 \mathrm{~km}$. The maximum density in the center of the star reaches $\rho_{c}=0.78 \mathrm{fm}^{-3}$ which corresponds to about five times normal nuclear density. Increasing the hyperon-hyperon interactions results in a lower maximum neutron star mass. A second stable solution appears for a range of parameters $\left(0.78>g_{\sigma^{*}} / g_{\sigma N}>0.71\right)$ constituting a third family of compact stars 26]. It is located beyond white dwarfs and ordinary neutron stars, with similar masses as predicted for neutron stars but with considerably smaller radii. The new solution originates from the phase transition to hypermatter. Compact stars belonging to this third family contain a pure core of deeply bound hypermatter consisting of about equal amounts of nucleons, $\Lambda$, and $\Xi$. The central baryonic densities of these cores are quite high, between $1.1 \mathrm{fm}^{-3}<\rho_{c}<2.0 \mathrm{fm}^{-3}$. The characteristic radius ranges from $8.6 \mathrm{~km}<R<11.6$ $\mathrm{km}$ (see Fig. 3), which is considerably smaller than for ordinary neutron stars. Therefore, the measurement of two neutron stars with similar masses but distinctly different radii will serve as a unique signal for the existence of neutron star twins. The possibility of neutron star twins and a third family of compact stars has been raised earlier in connection with pion condensation and quark stars [27], and more recently for the phase transition to strange quark matter within the MIT bag model [28] and perturbative QCD [29], and for kaon condensation [30]. If the hyperon-hyperon interaction is increased to $g_{\sigma^{*}} / g_{\sigma N} \geq 0.8$, the two separate solutions disappear. The neutron star mass rises continuously with energy density. For self-bound hyperon stars $\left(g_{\sigma^{*}} / g_{\sigma N}=1.0\right)$, we calculate radii of $4.2 \mathrm{~km}<R<7.3 \mathrm{~km}$. Hyperon stars can have radii as small as $4.2 \mathrm{~km}$ for compact object with masses as low as $M \approx 0.05 M_{\odot}$. The core is solely composed of hypermatter which is surrounded by a halo of nuclei and electrons. If one neglects the outer crust of these self-bound hyperon stars the corresponding curve starts from the origin and an upper boundary for the radii exists of $R<7.2 \mathrm{~km}$ (see Fig. 3, curve labeled 'no crust'). At first glance, the mass-radius relations as discussed here are looking strikingly similar to the ones proposed for strange (quark) stars [31,32]. Strange stars are built of absolutely stable strange quark matter and can have smaller radii than normal neutron stars. Nevertheless, the maximum mass and radius for strange stars is close to the one for an ordinary neutron star, $M_{\max }=1.5-2 M_{\odot}$ with $R \approx 10 \mathrm{~km}$ when using the MIT bag model [31]. Within the Nambu-Jona-Lasinio model, these values are a little bit smaller, $M_{\max }=1.23 M_{\odot}$ with $R \approx 8 \mathrm{~km}$ [32]. Hyperon stars, the hadronic counterparts of strange stars (as derived in the model used here) have extreme nuclear properties. They reach central baryonic densities of up to $\rho_{c}=2.1 \mathrm{fm}^{-3}$ for the most massive objects, where effects from the hadronic substructure will get important. The region, where hadronic equation of states are applicable, might in fact be rather small due to large $N_{c}$ arguments [29]. Hyperon matter can be transformed to strange quark matter by strong interactions, as they have similar strangeness fraction. Then, hyperon stars can form a doorway state for the formation of strange stars as no strange quark matter seed is needed [31].

The detection of compact stars with small radii combined with small masses $\left(M \approx M_{\odot}\right.$ or below) would signal the existence of a novel form of matter, be it strange matter or hypermatter, which does not need to be absolutely stable. Recently, the radius of the isolated neutron star RX J185635-3754 has been extracted by various groups [33]. Using the new Chandra spectra, the radius for a black-body emitter turns out to be only $R_{\infty}=6$ $\mathrm{km}$. If such a small radius is confirmed, it would signal the existence of hypercompact stars. Nevertheless, effects of an atmosphere can increase that value up to $R_{\infty}=15 \pm 3 \mathrm{~km}$ [33].

The conversion of a neutron star to a hyperon star should be a dynamical process, namely a nonspherical collapse which approximately conserves the number of baryons. Figure depicts the baryon number as a function of the gravitational mass of the compact stars. Note, that for a fixed baryon number, the twin star is energetically favored compared to ordinary neutron stars. The mass difference from an ordinary neutron star to its twin is about $0.03 M_{\odot}$ which corresponds to a conversion energy of about $0.5 \times 10^{53} \mathrm{erg}$. Therefore, the collapse to a twin star might have similar properties as a super- or hypernova collapse [34. The additional release of energy due to the formation of a hyperon star in a supernova event will generate a second energetic shock front in addition to the standard prompt shock (similar to strange star formation as discussed in [35]). The conversion to hyperon matter in compact stars will contain an interplay of astrophysical observables, such as the spinup ef- 


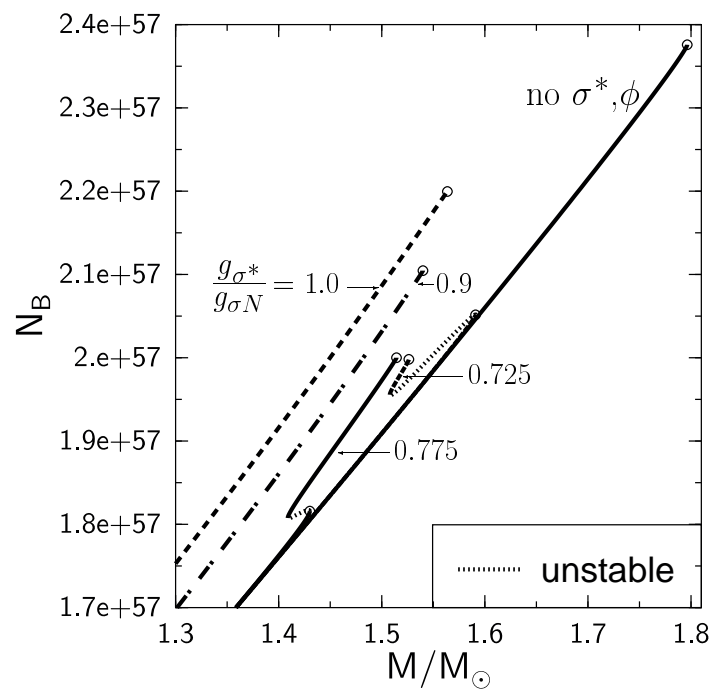

FIG. 4. The total baryon number $N_{B}$ versus the gravitational mass $M / M_{\odot}$.

fect [36], the emission of gravitational waves [37], and the emission of a $\gamma$-ray burst [38], as proposed for the conversion to deconfined matter. The emitted gravitational waves might be a relevant source for LIGO, VIRGO and GEO600 39].

We thank Klaus Schertler and Burkhard Kämpfer for fruitful discussions. JSB thanks RIKEN BNL and Brookhaven National Laboratory for their kind hospitality. $\mathrm{MH}$ thanks the Hessische Landesgraduiertenförderung for their support. This work is also supported in part by the Gesellschaft für Schwerionenforschung, the Deutsche Forschungsgemeinschaft via Graduiertenkolleg Theoretische und Experimentelle Schwerionenphysik, the Bundesministerium für Bildung und Forschung and the U.S. Department of Energy under Contract No. DE-FG-02-93ER-40764.

[1] N. K. Glendenning, Compact Stars, Nuclear Physics, Particle Physics, and General Relativity (Springer, New York, 2000).

[2] S. Balberg and A. Gal, Nucl. Phys. A625, 435 (1997).

[3] S. Pal, M. Hanauske, I. Zakout, H. Stöcker, W. Greiner, Phys. Rev. C 60, 015802 (1999).

[4] R. Knorren, M. Prakash, P.J. Ellis, Phys. Rev. C 52, 3470 (1995).

[5] J. Schaffner and I.N. Mishustin, Phys. Rev. C 53, 1416 (1996).

[6] H. Huber, F. Weber, M.K. Weigel, C. Schaab, Int. J. Mod. Phys. E7, 301 (1998).

[7] M. Baldo, G.F. Burgio, H.J. Schulze, Phys. Rev. C 61, 055801 (2000).

[8] I. Vidana, A. Polls, A. Ramos, L. Engvik, M. HjorthJensen, Phys. Rev. C 62, 035801 (2000).
[9] M. Hanauske, D. Zschiesche, S. Pal, S. Schramm, H. Stöcker, W. Greiner, Astrophys. J. 537, 958 (2000).

[10] D.J. Millener, C.B. Dover, A. Gal, Phys. Rev. C 38, 2700 (1988).

[11] C.J. Batty, E. Friedman, A. Gal, Prog. Theor. Phys. Suppl. 117, 227 (1994).

[12] S. Bart et al., Phys. Rev. Lett. 83, 5238 (1999).

[13] C.B. Dover and A. Gal, Ann. Phys. (N.Y.) 146, 309 (1983).

[14] T. Fukuda et al., Phys. Rev. C 58, 1306 (1998).

[15] P. Khaustov et al., Phys. Rev. C 61, 054603 (2000).

[16] J.K. Ahn et al., Phys. Rev. Lett. 87, 132504 (2001).

[17] H. Takahashi et al., Phys. Rev. Lett. 87, 212502 (2001).

[18] I.N. Filikhin and A. Gal, Phys. Rev. C 65, 041001 (2002).

[19] V.G.J. Stoks and T.A. Rijken, Phys. Rev. C 59, 3009 (1999).

[20] J. Schaffner-Bielich and A. Gal, Phys. Rev. C 62, 034311 (2000).

[21] J. Schaffner, C.B. Dover, A. Gal, C. Greiner, H. Stöcker, Phys. Rev. Lett. 71, 1328 (1993); J. Schaffner, C.B. Dover, A. Gal, D.J. Millener, C. Greiner, H. Stöcker, Ann. Phys. (N.Y.) 235, 35 (1994).

[22] J. Schaffner-Bielich, R. Mattiello, H. Sorge, Phys. Rev. Lett. 84, 4305 (2000).

[23] M. Rufa, J. Schaffner, J. Maruhn, H. Stöcker, W. Greiner, P.G. Reinhard, Phys. Rev. C 42, 2469 (1990).

[24] N.K. Glendenning and S.A. Moszkowski, Phys. Rev. Lett. 67, 2414 (1991).

[25] A.R. Bodmer, Phys. Rev. D 4, 1601 (1971).

[26] U.H. Gerlach, Phys. Rev. 172, 1325 (1968).

[27] B. Kämpfer, J. Phys. A14, L471 (1981); Phys. Lett. 101B, 366 (1981).

[28] N.K. Glendenning and C. Kettner, Astron. Astrophys. 353, L9 (2000); K. Schertler, C. Greiner, J. SchaffnerBielich, M.H. Thoma, Nucl. Phys. A 677, 463 (2000).

[29] E.S. Fraga, R.D. Pisarski, J. Schaffner-Bielich, Phys. Rev. D 63, 121702 (2001); Nucl. Phys. A702, 217 (2002).

[30] S. Banik and D. Bandyopadhyay, Phys. Rev. C 64, 055805 (2001).

[31] P. Haensel, J.L. Zdunik, R. Schaeffer, Astron. Astrophys. 160, 121 (1986); C. Alcock, E. Farhi, A. Olinto, Astrophys. J. 310, 261 (1986).

[32] M. Hanauske, L.M. Satarov, I.N. Mishustin, H. Stöcker, W. Greiner, Phys. Rev. D 64, 043005 (2001).

[33] J.A. Pons, F.M. Walter, J.M. Lattimer, M. Prakash, R. Neuhäuser, P. An, Astrophys. J. 564, 981 (2002); J.J. Drake et al., Astrophys. J. 572, 996 (2002); F.M. Walter and J. Lattimer, Astrophys. J. 576, L145 (2002).

[34] B. Kämpfer, Astrophys. Space Science 93, 185 (1983).

[35] O.G. Benvenuto and J.E. Horvath, Phys. Rev. Lett. 63, 716 (1989).

[36] N.K. Glendenning, S. Pei, F. Weber, Phys. Rev. Lett. 79, 1603 (1997).

[37] K.S. Cheng and Z.G. Dai, Astrophys. J. 492, 281 (1998).

[38] C. Alcock, E. Farhi, A. Olinto, Phys. Rev. Lett. 57, 2088 (1986); A.V. Olinto, Phys. Lett. B 192, 71 (1987); K.S. Cheng and Z.G. Dai, Phys. Rev. Lett. 77, 1210 (1996); ibid., 80, 18 (1998).

[39] B.J. Owen, L. Lindblom, C. Cutler, B.F. Schutz, A. Vecchio, N. Andersson, Phys. Rev. D 58, 084020 (1998). 